\title{
TOPICS IN THE THEORY OF ABELIAN GROUPS. I DIVISIBILITY OF HOMOMORPHISMS
}

\section{HASSLER WHITNEY}

1. Introduction. The theory of character groups of Abelian groups has in recent years become of great importance, especially through applications to topology and algebra. The character group of $G$ is the group $H$ of homomorphisms of $G$ into the real numbers mod 1 . Extending this, we may consider the group $H=\operatorname{Hom}(G, Z)$ of homomorphisms of $G$ into a third group $Z$, or more generally, a "pairing" of groups $H$ and $G$ into $Z$ : a multiplication $h \cdot g=z$, satisfying both distributive laws.

Of course the duality theorems for character groups will not hold in the more general cases; but, under certain conditions, substitutes may hold. We expect in later notes to give various facts about pairings, and the closely associated problem of divisibility by integers. In the present note, we answer the question of when a homomorphism of $G$ into $Z$ is divisible by an integer $m$; this has an immediate application to a theorem in combinatorial topology.

2. Divisibility theorems, discrete groups. We use the following notations for a group $X$ and an integer $m$.

$$
\begin{aligned}
& m X=\text { all } m x, \quad x \in X, \\
& { }_{m} X=\text { all } x \in X \text { such that } m x=0 .
\end{aligned}
$$

Note that $x \in m X$ means that $x$ is divisible by $m$.

In the theorems below, we use

$$
H=\operatorname{Hom}(G, Z) \text {. }
$$

Let $H^{\prime}\left(G^{\prime}\right)$ denote all $h(g), h \in H^{\prime}, g \in G^{\prime}$.

Lemma 1. Suppose $G^{\prime}$ is a subgroup of $G$,

$$
h \in H, \quad h^{\prime} \in \operatorname{Hom}\left(G^{\prime}, Z\right) .
$$

Then $h^{\prime}$ can be extended over $G$ so that $h=m h^{\prime}$ in $G^{\prime}$ if and only if:

( $\alpha) h(G) \subset m Z$.

(B) $h^{\prime}(m g)=h(g)$ if $m g \in G^{\prime}$.

( $\gamma$ ) If $m^{\prime}$ is a divisor of $m$, then $h^{\prime}\left(m^{\prime} g\right) \in m^{\prime} Z$ if $m^{\prime} g \in G^{\prime}$.

The necessity of the conditions is clear. We prove the sufficiency first for the case

Received by the editors November 19, 1943. 


$$
m=p^{n}, \quad p \text { prime. }
$$

To begin with, extend $h^{\prime}$ over $m G+G^{\prime}$ by setting

$$
h^{\prime}\left(m g+g^{\prime}\right)=h(g)+h^{\prime}\left(g^{\prime}\right) \quad\left(g^{\prime} \in G^{\prime}\right) .
$$

This definition is unique. For if $m g+g^{\prime}=m g_{1}+g_{1}^{\prime}$, then $m\left(g_{1}-g\right) \in G^{\prime}$, and $(\beta)$ gives

$0=h^{\prime}(0)=h^{\prime}\left[m\left(g_{1}-g\right)+\left(g_{1}^{\prime}-g^{\prime}\right)\right]=h\left(g_{1}-g\right)+h^{\prime}\left(g_{1}^{\prime}-g^{\prime}\right)$,

so that

$$
h(g)+h^{\prime}\left(g^{\prime}\right)=h\left(g_{1}\right)+h^{\prime}\left(g_{1}^{\prime}\right) .
$$

Clearly $(\beta)$ holds in $m G+G^{\prime}$. To prove $(\gamma)$, suppose $m=m^{\prime} m^{\prime \prime}$. Take any

$$
m^{\prime} g=m g_{1}+g^{\prime} \in m G+G^{\prime} .
$$

Then $m^{\prime}\left(g-m^{\prime \prime} g_{1}\right)=g^{\prime} \in G^{\prime}$, and hence, using (2), $(\alpha)$, and $(\gamma)$ in $G^{\prime}$, we find

$$
h^{\prime}\left(m^{\prime} g\right)=h\left(g_{1}\right)+h^{\prime}\left[m^{\prime}\left(g-m^{\prime \prime} g_{1}\right)\right] \in m^{\prime} Z .
$$

Let $S$ be the set of all pairs $\left(\phi, G^{\prime \prime}\right)$, where

$$
\begin{gathered}
m G+G^{\prime} \subset G^{\prime \prime} \subset G, \\
\phi \in \operatorname{Hom}\left(G^{\prime \prime}, Z\right), \quad \phi=h^{\prime} \text { in } m G+G^{\prime},
\end{gathered}
$$

and such that $(\beta)$ and $(\gamma)$ hold with $\phi$ and $G^{\prime \prime}$ in place of $h^{\prime}$ and $G^{\prime}$. Partially order these by setting

$$
\left(\phi_{1}, G_{1}\right) \prec\left(\phi_{2}, G_{2}\right) \text { if } G_{1} \subset G_{2} \text { and } \phi_{2}=\phi_{1} \text { in } G_{1},
$$

that is, $\phi_{2}$ is an extension of $\phi_{1}$. By ${ }^{1}$ Zorn's lemma, there is a maximal simply ordered set $S^{\prime}$ of elements of $S$. Let $G_{0}$ be the set of all $g$ in some $G^{\prime \prime}$ with $\left(\phi, G^{\prime \prime}\right) \in S^{\prime}$, and set $\phi_{0}(g)=\phi(g)$ for such a $\left(\phi, G^{\prime \prime}\right)$. Clearly $G_{0}$ is a subgroup of $G, \phi_{0}(g)$ is independent of the $\left(\phi, G^{\prime \prime}\right)$ chosen, and $\left(\phi_{0}, G_{0}\right) \in S^{\prime}$; hence it is the last element in $S^{\prime}$, and there is no later element in $S$. Therefore $\phi_{0}$ cannot be further extended in $G$. To prove the lemma for $m=p^{n}$, it is sufficient to show that $G_{0}=G$; for $\phi_{0}=h^{\prime}$ in $G^{\prime}$ by hypothesis, and $(\beta)$ gives

$$
m \phi_{0}(g)=\phi_{0}(m g)=h(g), \quad m \phi_{0}=h .
$$

Suppose $G_{0} \neq G$. Then choose an element $g_{1} \notin G_{0}$ such that for a largest possible divisor $m_{1}$ of $m, g_{1} \in m_{1} G$. Since $m G \subset G_{0}$, it follows

1 Compare Remark 2 in $\$ 3$. 
that $m_{1}<m$, and $m_{1}=p^{\mu}, \mu<n$. Choose $g_{3}$ and $g_{2}$ so that

$$
g_{1}=m_{1} g_{3}, \quad g_{2}=p g_{1}=p m_{1} g_{3} ;
$$

then $g_{2} \in G_{0}$. Set $m_{2}=p^{n-\mu-1}$; then $p m_{1} m_{2}=m$. Since $\phi_{0}=h^{\prime}$ in $m G+G^{\prime}$, (2) gives

$$
m_{2} \phi_{0}\left(g_{2}\right)=\phi_{0}\left(p m_{1} m_{2} g_{3}\right)=h\left(g_{3}\right) .
$$

Since $g_{2}=p m_{1} g_{3} \in G_{0}$, we may choose $z_{2}$ by $(\gamma)$ so that

$$
\phi_{0}\left(g_{2}\right)=p m_{1} z_{2} \text {. }
$$

Let $G_{1}$ be the subgroup of $G$ generated by $G_{0}$ and $g_{1}$. Since $p$ is prime, and $p g_{1}$ is in $G_{0}$ while $g_{1}$ is not, $a g_{1} \in G_{0}$ if and only if $a \equiv 0$ $(\bmod p)$. Extend $\phi_{0}$ through $G_{1}$ by setting

$$
\phi_{0}\left(a g_{1}+g\right)=a m_{1} z_{2}+\phi_{0}(g) \quad\left(a \text { integral, } g \in G_{0}\right) \text {. }
$$

To show that this is unique, suppose

Then

$$
a g_{1}+g=b g_{1}+g^{\prime}, \quad g, g^{\prime} \in G_{0} \text {. }
$$

$$
(a-b) g_{1}=g^{\prime}-g \in G_{0}, \quad a-b=k p,
$$

and

$$
\begin{aligned}
{\left[a m_{1} z_{2}+\phi_{0}(g)\right]-\left[b m_{1} z_{2}+\phi_{0}\left(g^{\prime}\right)\right] } & =k p m_{1} z_{2}+\phi_{0}\left(g-g^{\prime}\right) \\
& =k \phi_{0}\left(g_{2}\right)-\phi_{0}\left(k p g_{1}\right) \\
& =\phi_{0}\left(k g_{2}-k g_{2}\right)=0,
\end{aligned}
$$

as required. Clearly $\phi_{0}$ is now a homomorphism in $G_{1}$.

Since $m G \subset G_{0},(\beta)$ holds in $G_{1}$. To prove $(\gamma)$, take any

$$
m^{\prime} g=a g_{1}+g_{0} \in G_{1} \quad\left(g_{0} \in G_{0}\right) .
$$

If $m^{\prime} g \in G_{0}$, then $\phi_{0}\left(m^{\prime} g\right) \in m^{\prime} Z$, since $(\gamma)$ holds in $G_{0}$. Suppose it is not. By the choice of $g_{1}$ and $m_{1}, m^{\prime} \leqq m_{1}$; hence for some $m^{\prime \prime}, m^{\prime} m^{\prime \prime}=m_{1}$. Now by (3),

$$
g_{0}=m^{\prime} g-a m_{1} g_{3}=m^{\prime}\left(g-a m^{\prime \prime} g_{3}\right),
$$

and by $(\gamma)$ in $G_{0}, \phi_{0}\left(g_{0}\right)=m^{\prime} z_{0}$ for some $z_{0}$. Now by (6),

$$
\phi_{0}\left(m^{\prime} g\right)=a m_{1} z_{2}+\phi_{0}\left(g_{0}\right)=m^{\prime}\left(a m^{\prime \prime} z_{2}+z_{0}\right) \text {, }
$$

as required.

We have now shown that $\left(\phi_{0}, G_{1}\right) \in S$. Since $\phi_{0}$ has been extended from $G_{0},\left(\phi_{0}, G_{0}\right)<\left(\phi_{0}, G_{1}\right)$. But this contradicts the fact that $S^{\prime}$ was maximal. It follows that $G_{0}=G$, and the lemma is proved for the case $m=p^{n}$. 
For a general $m$, we shall use induction on the number $\rho(m)$ of distinct prime divisors of $m$. Say $m=m_{1} m_{2},\left(m_{1}, m_{2}\right)=1, \rho\left(m_{1}\right)$ and $\rho\left(m_{2}\right)$ being less than $\rho(m)$. Set

$$
h_{1}=m_{2} h^{\prime}, \quad h_{2}=m_{1} h^{\prime}, \quad \text { in } G^{\prime} .
$$

Then the conditions of the lemma hold for both $h_{1}$ and $h_{2}$, with $m_{1}$ and $m_{2}$ respectively. Property $(\alpha)$ for $m_{1}$ and $m_{2}$ is clear. To prove $(\beta)$, we have

$$
h_{1}\left(m_{1} g\right)=m_{2} h^{\prime}\left(m_{1} g\right)=h^{\prime}(m g)=h(g) .
$$

To prove $(\gamma)$, suppose $m_{1}^{\prime}$ is a divisor of $m_{1}$ and $m_{1}^{\prime} g \in G^{\prime}$; then $m_{1}^{\prime}$ is a divisor of $m$, and $(\gamma)$ for $h^{\prime}$ gives

$$
h_{1}\left(m_{1}^{\prime} g\right)=m_{2} h^{\prime}\left(m_{1}^{\prime} g\right) \in m_{2}\left(m_{1}^{\prime} Z\right) \subset m_{1}^{\prime} Z .
$$

Therefore $h_{1}$ and $h_{2}$ may be extended over $G$, so that

$$
h=m_{1} h_{1}=m_{2} h_{2} \text {. }
$$

Now choose integers $r, s$ so that $r m_{1}+s m_{2}=1$. Then

$$
\begin{aligned}
r h_{2}+s h_{1} & =\left(r m_{1}+s m_{2}\right) h^{\prime}=h^{\prime} \text { in } G^{\prime}, \\
m\left(r h_{2}+s h_{1}\right) & =r m_{1} m_{2} h_{2}+s m_{2} m_{1} h_{1}=h \text { in } G,
\end{aligned}
$$

so that $h^{\prime}=r h_{2}+s h_{1}$ is the required homomorphism.

THEOREM 1. For any integer $m, h \in m H$ if and only if

(a) $h(G) \subset m Z$,

(b) $h\left({ }_{m} G\right)=0$.

Let $G^{\prime}$ be the identity alone, and set $h^{\prime}(0)=0$. We need merely prove $(\beta)$ and $(\gamma)$ of Lemma 1 . Clearly $(\beta)$ reduces to $(b) ;(\gamma)$ is trivial.

3. Divisibility properties, topological groups. We shall consider only topological groups with the following property:

(P) For each integer $m>0$, the mapping $m g$ of $G$ into itself is interior, that is, maps open sets into open sets.

An equivalent statement is the following: For every neighborhood $U$ of 0 in $G$ and every $m>0$ there is a neighborhood $U^{\prime}$ of 0 such that $U^{\prime} \subset m U$; that is, all sufficiently small elements of $G$ are divisible by $m$, a solution lying in $U$. The property holds in the commonly used groups.

REMARK 1. It follows that $m G$ is open in $G(m>0)$; hence, for any subgroup $G^{\prime}, m G+G^{\prime}$ is open in $G$.

EXAMPLE. If $G$ is the group of dyadic rational numbers, using the 
topology of the real numbers, and $m=3$, then $3 G$ contains no element of the form $1 / 2^{n}$. Thus $G$ does not satisfy (P).

LEMma 2. Let $G$ and $Z$ be topological groups, and let (P) hold in $G$. Let $h^{\prime}$ be a homomorphism of $G$ into $Z$, and set $h=m h^{\prime}$. Then $h^{\prime}$ is continuous if and only if $h$ is.

That continuity of $h$ follows from that of $h^{\prime}$ is simple (and does not use (P)). Suppose $h$ is continuous. Given any neighborhood $V$ of 0 in $Z$, choose a neighborhood $U_{1}$ of 0 in $G$ so that $h\left(U_{1}\right) \subset V$, and choose $U \subset m U_{1}$. Now take any $g \in U$. Then $g=m g_{1}, g_{1} \in U_{1}$, and $h^{\prime}(g)$ $=h\left(g_{1}\right) \in V$, proving that $h^{\prime}$ is continuous.

By Hom $(G, Z)$ we shall now mean the group of continuous homomorphisms of $G$ into $Z$. We do not need to consider a topology in $H$ here.

THEOREM 2. If $G$ and $Z$ are topological groups, and (P) holds in $G$, then Lemma 2 and Theorem 1 hold.

Using the former proofs, we need merely prove the continuity of $h^{\prime}$. But this follows from the continuity of $h$ and the last lemma.

REMARK 2. Since $m G$ is open in $G$ and $h^{\prime}$ is first extended over $m G$, if the proof in Lemma 1 is given in the equivalent form of extending $h^{\prime}$ over larger and larger subgroups of $G$ (as from $G_{0}$ to $G_{1}$ in the proof given), only a finite number of steps will be required in the case of most groups $G$.

4. On the complete resolution of $H$ by $G$. We use the following definitions and facts. ${ }^{2}$ If $H, G$ are paired into $Z,\left(H, G^{\prime}\right)$ (the nullifier or annihilator of $G^{\prime}$ in $\left.H\right)$ is the set of all $h$ with $h \cdot G^{\prime}=0$. If $\left(H, G^{\prime}\right)=0$, then $G^{\prime}$ resolves $H$. (Then if $h_{1} \neq h_{2}$, there is an element $g \in G^{\prime}$ with $h_{1} \cdot g \neq h_{2} \cdot g$.) If $\left(H,{ }_{m} G^{\prime}\right)=m H, G^{\prime} m$-resolves $H$. (Then if $h$ is not divisible by $m$, there is an element $g \in{ }_{m} G^{\prime}$ such that $h \cdot g \neq 0$.) "Resolves" and "0-resolves" are equivalent. If $G^{\prime} m$-resolves $H$ for all integers $m \geqq 0, G^{\prime}$ resolves $H$ completely. $Z$ is completely divisible if $m Z=Z$ for each integer $m \neq 0$. The main application of the Pontrjagin duality theory to topology lies in the fact ${ }^{2}$ that any group resolves completely and is resolved completely by its character group.

Theorem 3. If $Z$ is completely divisible, then $G$ resolves $H$ $=\operatorname{Hom}(G, Z)$ completely.

2 Compare H. Whitney, On matrices of integers and combinatorial topology, Duke Math. J. vol. 3 (1937) pp. 35-45. The main statement in footnote 8 of this paper is not true. 
If the groups are topological, we assume $G$ satisfies (P).

If $h \in m H$, say $h=m h^{\prime}$, then $m g=0$ implies

$$
h(g)=h^{\prime}(m g)=h^{\prime}(0)=0,
$$

and $h \in\left(H,{ }_{m} G\right)$. Conversely, if $h \in\left(H,{ }_{m} G\right)$, that is, $h\left({ }_{m} G\right)=0$, Theorem 1(or Theorem 2) gives $h \in m H$.

5. Application to combinatorial topology. Let $H^{r}(K, X)$ and $H_{r}(K, X)$ denote the $r$ th homology and cohomology groups, respectively, of $K$, with the coefficient group $X$.

THEOREM 4. Let $Z$ be completely divisible. Then for any $G$, and $H=\operatorname{Hom}(G, Z)$, we have the isomorphisms

$$
\begin{aligned}
& H_{r}(K, H) \approx \operatorname{Hom}\left[H^{r}(K, G), Z\right], \\
& H^{r}(K, H) \approx \operatorname{Hom}\left[H_{r}(K, G), Z\right] .
\end{aligned}
$$

If $G$ and $Z$ are topological, we assume $G$ satisfies (P).

This is Theorem 8 of Whitney, loc. cit., with the hypothesis weakened; it is essentially the Pontrjagin Duality Theorem, slightly generalized. For the proof, we need merely apply Theorem 3 to obtain the remainder of the hypothesis.

HARVARD UNIVERSITY 